\title{
A Survey of Equal Sums of Like Powers
}

\author{
By L. J. Lander, T. R. Parkin and J. L. Selfridge
}

Introduction. The Diophantine equation

$$
x_{1}{ }^{k}+x_{2}{ }^{k}+\cdots+x_{m}{ }^{k}=y_{1}{ }^{k}+y_{2}{ }^{k}+\cdots+y_{n}{ }^{k}, \quad 1 \leq m \leq n,
$$

has been studied by numerous mathematicians for many years and by various methods [1], [2]. We recently conducted a series of computer searches using the CDC 6600 to identify the sets of parameters $k, m, n$ for which solutions exist and to find the least solutions for certain sets. This paper outlines the results of the computation, notes some previously published results, and concludes with a table showing, for various values of $k$ and $m$, the least $n$ for which a solution to (1) is known.

We restrict our attention to $k \leq 10$. We assume that the $x_{i}$ and $y_{j}$ are positive integers and $x_{i} \neq y_{j}$. We do not distinguish between solutions which differ only in that the $x_{i}$ or $y_{j}$ are rearranged. We will refer to (1) as (k.m.n) and say that a primitive solution to $(k . m . n)$ is one in which no integer $>1$ divides all the numbers $x_{1}, x_{2}, \cdots, x_{m}, y_{1}, y_{2}, \cdots, y_{n}$. Putting

$$
z=\sum_{1}^{m} x_{i}{ }^{k}=\sum_{1}^{n} y_{j}{ }^{k},
$$

we order the primitive solutions according to the magnitude of $z$ and denote the $r$ th primitive solution to $(k . m . n)$ by $(k . m . n)_{r}$. Where we refer to the range covered in a search for solutions, we mean the upper limit on $z$. The notation $\left(x_{1}, x_{2}, \cdots, x_{m}\right)^{k}$ $=\left(y_{1}, y_{2}, \cdots, y_{n}\right)^{k}$ means $\sum_{1}^{m} x_{i}{ }^{k}=\sum_{1}^{n} y_{j}{ }^{k}$. Any parametric solution discussed does not include all solutions unless otherwise stated.

Squares and Cubes. For $k=2$ the general solution of the Pythagorean equation (2. 1. 2) is well known [3]. Many solutions in small integers and various parametric solutions have been given for (2.1.n) with $n \geq 3$. The general solution of $(2.2 .2)$ is known [4]. Solutions to (2.2.n) with $n \geq 3$ and (2.m.n) with $m \geq 3$ are numerous.

The impossibility of solving ( $k .1 .2)$ with $k \geq 3$ is Fermat's last theorem, which has been established for $k \leq 25000$ [5]. The general solution of (3.1.3) in rationals is attributed to Euler and Vieta [6] and also produces all solutions to (3.2.2) if the arguments are properly chosen. There are many solutions in small integers and various parametric solutions to (3.1.n) with $n \geq 4$ and to (3.m.n) with $m \geq 2$ [7].

\section{Fourth Powers.}

(4. 1. $n$ )-For $n=3$, no solution is known. M. Ward [8] developed congruential constraints which, together with some hand computing, allowed him to show that $x^{4}=y_{1}{ }^{4}+y_{2}{ }^{4}+y_{3}{ }^{4}$ has no solution if $x \leq 10,000$. The authors extended the search on the computer using a similar method and verified that there is no solution for $x \leq 220,000$. Ward showed that if $x^{4}=y_{1}{ }^{4}+y_{2}{ }^{4}+y_{3}{ }^{4}$ is a primitive solution, it may be assumed that $x, y_{1} \equiv 1(\bmod 2), y_{2}, y_{3} \equiv 0(\bmod 8)$ and either $x-y_{1}$ or $x+y_{1}$

Received September 30, 1966. Revised December 12, 1966. 
is $\equiv 0(\bmod 1024)$. Also $x \not \equiv 0(\bmod 5)$ or else all $y_{i}$ would be $\equiv 0(\bmod 5)$ since $u^{4}$ $\equiv 0$ or 1 according as $u \equiv 0$ or $u \neq \equiv 0(\bmod 5)$. The computer program generated all numbers $M=\left(x^{4}-y_{1}^{4}\right) / 2048$ with $0<y_{1}<x, x$ prime to 10 and $y_{1} \equiv \pm x(\bmod$ 1024). Tests were applied to $M=\left(y_{2} / 8\right)^{4}+\left(y_{3} / 8\right)^{4}$ to reject cases in which a solution would not be primitive or $M$ could not be the sum of two biquadrates. If $M$ passed all the tests, its decomposition was attempted by trial using addition of entries in a stored table of biquadrates (27500 entries for $x \leq 220,000=8 \cdot 27500)$. The tests were:

(1) $M$ must be $\equiv 0,1$ or $2(\bmod 16)$ and $(\bmod 5)$;

(2) $M$ must not be $\equiv 7,8$ or $11(\bmod 13)$ and must not be $\equiv 4,5,6,9,13,22$ or $28(\bmod 29)$;

(3) $x$ and $y_{1}$ must not both be divisible by an odd prime $p \equiv 3,5$ or $7(\bmod 8)$ for if so, $p^{4}$ divides $M, p$ divides $y_{2}$ and $y_{3}$ and the solution is not primitive;

(4) $M$ must not have a factor $p$ where $p$ is an odd prime not $\equiv 1(\bmod 8)$ unless $p^{4}$ also divides $M$. In this case $p$ divides $y^{2}$ and $y^{3}$, and in the decomposition by trial $M$ can be replaced by $M / p^{4}$ (here tests were made only for $p<100$ ).

Of approximately $19,200,000$ initial values of $M$, only 22,400 required the trial decomposition.

TABLE I

Primitive solutions of (4. 1. 4) for $z \leq(8002)^{4}$ $z=x_{1}{ }^{4}=\sum_{1}^{4} y_{j}{ }^{4}$

\begin{tabular}{|c|c|c|c|c|c|c|}
\hline$i$ & $x_{1}$ & $y_{1}$ & $y_{2}$ & $y_{3}$ & $y_{4}$ & Ref. \\
\hline 1 & 353 & 30 & 120 & 272 & 315 & [9] \\
\hline 2 & 651 & 240 & 340 & 430 & 599 & [34] \\
\hline 3 & 2487 & 435 & 710 & 1384 & 2420 & {$[10]$} \\
\hline 4 & 2501 & 1130 & 1190 & 1432 & 2365 & {$[10]$} \\
\hline 5 & 2829 & 850 & 1010 & 1546 & 2745 & {$[10]$} \\
\hline 6 & 3723 & 2270 & 2345 & 2460 & 3152 & {$[10]$} \\
\hline 7 & 3973 & 350 & 1652 & 3230 & 3395 & {$[10]$} \\
\hline 8 & 4267 & 205 & 1060 & 2650 & 4094 & [10] \\
\hline 9 & 4333 & 1394 & 1750 & 3545 & 3670 & \\
\hline 10 & 4449 & 699 & 700 & 2840 & 4250 & \\
\hline 11 & 4949 & 380 & 1660 & 1880 & 4907 & \\
\hline 12 & 5281 & 1000 & 1120 & 3233 & 5080 & \\
\hline 13 & 5463 & 410 & 1412 & 3910 & 5055 & \\
\hline 14 & 5491 & $95 \tilde{5}$ & 1770 & 2634 & 5400 & [11] \\
\hline 15 & 5543 & 30 & 1680 & 3043 & 5400 & \\
\hline 16 & 5729 & 1354 & 1810 & 4355 & 5150 & \\
\hline 17 & 6167 & 542 & 2770 & 4280 & 5695 & \\
\hline 18 & 6609 & 50 & 885 & 5000 & 5984 & \\
\hline 19 & 6801 & 1490 & 3468 & 4790 & 6185 & \\
\hline 20 & 7101 & 1390 & 2850 & 5365 & 6368 & \\
\hline 21 & 7209 & 160 & 1345 & 2790 & 7166 & \\
\hline 22 & 7339 & 800 & 3052 & 5440 & 6635 & \\
\hline 23 & 7703 & 2230 & 3196 & 5620 & 6995 & \\
\hline
\end{tabular}


For $n=4$, R. Norrie [9] found the smallest solution $(353)^{4}=(30,120,272,315)^{4}$. J. O. Patterson [34] found (4.1.4) $)_{2}$ and J. Leech [10] found the next 6 primitive solutions on the EDSAC 2 computer. S. Brudno [11] gave another primitive solution, the 14 th in our Table I. The authors exhaustively searched the range $8002^{4}$ using Leech's method finding in all the 23 primitives listed in Table I. No parametric solution has been found for (4. 1.4) although the general solution is known for (3. 1.3) and a parametric solution (discussed later) is known for (5. 1. 5).

\section{TABLE II}

Primitive solutions of (4.2.2) for $7.5 \times 10^{15} \leq z \leq 5.3 \times 10^{16}$

$$
z=x_{1}{ }^{4}+x_{2}{ }^{4}=y_{1}{ }^{4}+y_{2}{ }^{4}
$$

\begin{tabular}{|c|c|c|c|c|c|c|}
\hline$i$ & $x_{1}$ & $x_{2}$ & $y_{1}$ & $y_{2}$ & & $z$ \\
\hline$* 32$ & 6262 & 8961 & 7234 & 8511 & 798564 & 4522300177 \\
\hline 33 & 5452 & 9733 & 7528 & 9029 & 985755 & 1363885937 \\
\hline 34 & 3401 & 10142 & 7054 & 9527 & 1071400 & 4223480497 \\
\hline 35 & 5277 & 10409 & 8103 & 9517 & 1251457 & 3616092402 \\
\hline 36 & 3779 & 10652 & 8332 & 9533 & 1307827 & 2245398097 \\
\hline 37 & 3644 & 11515 & 5960 & 11333 & 1775781 & 8522558321 \\
\hline 38 & 1525 & 12234 & 3550 & 12213 & 2240674 & 3733252161 \\
\hline$* * 39$ & 2903 & 12231 & 10203 & 10381 & 2245039 & 1640617602 \\
\hline 40 & 1149 & 12653 & 7809 & 12167 & 2563324 & 3495011682 \\
\hline 41 & 5121 & 13472 & 9153 & 12772 & 3362808 & 8414785537 \\
\hline 42 & 5526 & 13751 & 11022 & 12169 & $36 \quad 68751$ & 7059308977 \\
\hline 43 & 6470 & 14421 & 8171 & 14190 & 4500187 & 6412998081 \\
\hline 44 & 6496 & 14643 & 11379 & 13268 & 4775551 & $49900 \quad 03857$ \\
\hline 45 & 261 & 14861 & 8427 & 14461 & 4877442 & 7226631682 \\
\hline 46 & 581 & 15109 & 8461 & 14723 & 5211273 & 1140326882 \\
\hline
\end{tabular}

* For solutions to (4.2.2) for $i=1$ to 31 see Lander and Parkin [18].

** This solution was found by Euler [37].

For $n \geq 5$ there exist many solutions in small integers. (4. 1. 5) 1 is $(5)^{4}=$ $(2,2,3,4,4)^{4}$. Several parametric solutions to (4. 1. 5) are known due to E. Fauquembergue [12], C. Haldeman [13], and A. Martin [14].

(4. 2. $n)$-For $n=2$ the least solution is $(59,158)^{4}=(133,134)^{4}$. Euler [15] gave a two-parameter solution and A. Gérardin [16] gave an equivalent but simpler form of this solution. Several of the smaller primitive solutions were found by Euler, A. 
Werebrusow, and Leech [17] and a recent computer search by Lander and Parkin [18] extended the list of known primitives to 31 . More recently we have increased this to a total of 46 primitives by a complete search of the range $5.3 \times 10^{16}$ and the 15 new primitives are listed in Table II. The general solution is not known.

For $n \geq 3$ there are many small solutions. $(4.2 .3)_{1}$ is $(7,7)^{4}=(3,5,8)^{4}$. Several parametric solutions are known for (4. 2. 3) due to Gérardin [19] and F. Ferrari [20].

(4. m.n)-For $m \geq 3$, solutions in small integers are numerous. Parametric solutions to (4. 3. 3) were given by Gérardin [21] and Werebrusow [22]. (4. 3. 3) 1 is $(2,4,7)^{4}=(3,6,6)^{4}$.

\section{Fifth Powers.}

(5. 1. $n$ )-For $n=3$, no solution is known. Lander and Parkin [23], [24] found (5. 1. 4) $)_{1}$ to be $(144)^{5}=(27,84,110,133)^{5}$. This disproved Euler's conjecture [25] that $(k .1 . n)$ has no solution if $1<n<k$. No further primitive solutions to (5.1.4) exist in the range up to $765^{5}$.

For $n=5$, S. Sastry and S. Chowla [26] obtained a two-parameter solution yield . ing $(107)^{5}=(7,43,57,80,100)^{5}$ as its minimal primitive; this solution is $(5.1 .5)_{3}$. Lander and Parkin [24] found (5.1.5) 1 and (5.1.5) $)_{2}$ to be $(72)^{5}=(19,43,46,47,67)^{5}$ and $(94)^{5}=(21,23,37,79,84)^{5}$. More recently we searched the range up to $599^{5}$ and found in all the twelve primitive solutions given in Table III.

\section{TABLE III}

Primitive solutions of (5. 1. 5) for $z \leq 599^{5}$

$$
z=x_{1}{ }^{5}=\sum_{1}^{5} y_{j}{ }^{5}
$$

\begin{tabular}{r|r|rrrrr|r}
\hline$i$ & $x_{1}$ & $y_{1}$ & $y_{2}$ & $y_{3}$ & $y_{4}$ & $y_{5}$ & Ref. \\
\hline 1 & 72 & 19 & 43 & 46 & 47 & 67 & {$[24]$} \\
2 & 94 & 21 & 23 & 37 & 79 & 84 & {$[24]$} \\
3 & 107 & 7 & 43 & 57 & 80 & 100 & {$[26]$} \\
4 & 365 & 78 & 120 & 191 & 259 & 347 & \\
5 & 415 & 79 & 202 & 258 & 261 & 395 & \\
6 & 427 & 4 & 26 & 139 & 296 & 412 & \\
7 & 435 & 31 & 105 & 139 & 314 & 416 & \\
8 & 480 & 54 & 91 & 101 & 404 & 430 & \\
9 & 503 & 19 & 201 & 347 & 388 & 448 & \\
10 & 530 & 159 & 172 & 200 & 356 & 513 & \\
11 & 553 & 218 & 276 & 385 & 409 & 495 & \\
12 & 575 & 2 & 298 & 351 & 474 & 500 & \\
\hline
\end{tabular}


For $n \geq 6$ there are solutions in moderately small integers. $(5.1 .6)_{1}$ is $(12)^{\mathfrak{j}}=$ $(4,5,6,7,9,11)^{5}$ found by A. Martin [27]. The first eight primitive solutions to (5. 1.6) are given in [24]. $(5.1 .7)_{1}$ is $(23)^{5}=(1,7,8,14,15,18,20)^{5}$.

(5. 2. $n)$ - No solution is known for $n \leq 3$. An exhaustive search by the authors verified that there is no solution to (5.2.2) in the range up to $2.8 \times 10^{14}$ or to (5. 2. 3) in the range up to $8 \times 10^{12}$. Sastry's parametric solution for (5. 1. 5) mentioned above gives for certain values of its arguments solutions to (5. 2. 4), the smallest being $(12,38)^{5}=(5,13,25,37)^{5}$ which is $(5.2 .4)_{2}$. K. Subba Rao [28] found $(3,29)^{5}=(4,10,20,28)^{5}$ which is $(5.2 .4)_{1}$. Table IV lists the ten primitives which exist in the range up to $2 \times 10^{10}$.

\section{TABLE IV}

Primitive solutions of (5. 2. 4) for $z \leq 2 \times 10^{10}$

$$
z=\sum_{1}^{2} x_{j}{ }^{5}=\sum_{1}^{4} y_{j}{ }^{5}
$$

\begin{tabular}{|c|c|c|c|c|c|c|c|c|}
\hline$i$ & $x_{1}$ & $x_{2}$ & $y_{1}$ & $y_{2}$ & $y_{3}$ & $y_{4}$ & $z$ & Ref. \\
\hline 1 & 3 & 29 & 4 & 10 & 20 & 28 & 20511392 & {$[28]$} \\
\hline 2 & 12 & 38 & 5 & 13 & 25 & 37 & 79484000 & {$[26]$} \\
\hline 3 & 28 & 52 & 26 & 29 & 35 & 50 & 397414400 & \\
\hline 4 & 61 & 64 & 5 & 25 & 62 & 63 & 1918338125 & \\
\hline 5 & 16 & 85 & 6 & 50 & 53 & 82 & 4438101701 & \\
\hline 6 & 31 & 96 & 56 & 63 & 72 & 86 & 8182356127 & \\
\hline 7 & 14 & 99 & 44 & 58 & 67 & 94 & 9510438323 & \\
\hline 8 & 63 & 97 & 11 & 13 & 37 & 99 & 9579776800 & \\
\hline 9 & 25 & 106 & 48 & 57 & 76 & 100 & 13392021401 & \\
\hline 10 & 54 & 111 & 58 & 76 & 79 & 102 & 17309746575 & \\
\hline
\end{tabular}

For $n \geq 5$ there are solutions in moderately small integers; $(5.2 .5)_{1}$ is $(1,22)^{5}=$ $(4,5,7,16,21)^{5}$ due to Subba Rao [28]. We give the first six primitives for (5.2.5) in Table V.

(5. 3. $n$ - -The first solution known for $n=3$ was $(49,75,107)^{5}=(39,92,100)^{5}$

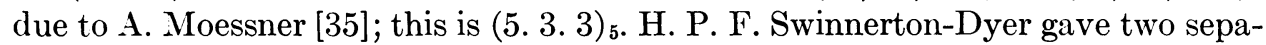
rate two-parameter solutions [36]. We give the 45 primitives in the range up to $8 \times$ $10^{12}$ in Table VI. For $n \geq 4$, solutions in small integers are plentiful. (5. 3.4) 1 is $(3,22,25)^{5}=(1,8,14,27)^{5}$ due to Subba Rao [28]. A two-parameter solution to (5. 3. 4) was given by G. Xeroudakes and A. Moessner [29].

(5. $m . n)$-If $m \geq 4$, there are many solutions in small integers. (5. 4. 4) $)_{1}$ is $(5,6,6,8)^{5}=(4,7,7,7)^{5}$ due to Subba Rao [28]. Several parametric solutions to (5. 4. 4) were found by Xeroudakes and Moessner [29]. The first triple coincidence of four fifth powers is $1479604544=(3,48,52,61)^{5}=(13,36,51,64)^{5}=(18,36,44,66)^{5}$. 
In the subsequent discussion we adopt a notation borrowed from the field of partitions, writing $x^{r}$ to signify the term $x$ repeated $r$ times in the expression in which it appears. Table VII uses this notation, giving $(k . m . n)_{1}$ where known and references solutions in other tables. Table VII also shows for certain (k.m.n) the range which has been searched on the computer exhaustively.

For the remainder of the equations $(k . m . n)$ which are discussed we note in the text only the limits searched, interesting features, and methods employed; specific solutions are given in Table VII.

\section{Sixth Powers.}

(6. 1. $n)-$ No solution is known for $n \leq 6$. We consider the cases of $n=6,7$ and 8 in descending order. To solve (6.1.8), $x^{6}=\sum_{1}^{8} y_{i}{ }^{6}$, note that $u^{6} \equiv 0$ or $1(\bmod 9)$ according as $u \equiv 0$ or $u \neq \equiv 0(\bmod 3)$. Then if $x \equiv 0(\bmod 3)$, all $y_{i} \equiv 0(\bmod 3)$ and the solution is not primitive. Therefore take $x$ and exactly one of the $y_{i}$ (say $y_{1}$ ) prime to 3 . Then $\left(x^{6}-y_{1}^{6}\right) / 3^{6}=\sum_{2}^{8}\left(y_{i} / 3\right)^{6}$ is an integer (which is true if and only if $y_{1} \equiv$ $\pm x(\bmod 243))$ to be decomposed by trial as the sum of 7 sixth powers. In Table VIII we give the 14 smallest primitives found by this method; $(6.1 .8)_{1}$ is $(251)^{6}=$ $(8,12,30,78,102,138,165,246)^{6}$.

\section{TABLE V}

Primitive solutions of (5. 2. 5) for $z \leq 2.8 \times 10^{8}$

$$
z=\sum_{1}^{2} x_{j}{ }^{5}=\sum_{1}^{5} y_{j}{ }^{5}
$$

\begin{tabular}{r|rr|rrrrr|r}
\hline$i$ & $x_{1}$ & $x_{2}$ & $y_{1}$ & $y_{2}$ & $y_{3}$ & $y_{4}$ & \multicolumn{1}{c}{$y_{5}$} & \multicolumn{2}{|c}{$z$} \\
\cline { 2 - 7 } & 1 & 22 & 4 & 5 & 7 & 16 & 21 & 5153633 \\
2 & 23 & 29 & 9 & 11 & 14 & 18 & 30 & 26947492 \\
3 & 16 & 38 & 10 & 14 & 26 & 31 & 33 & 80283744 \\
4 & 24 & 42 & 4 & 22 & 29 & 35 & 36 & 138653856 \\
5 & 30 & 44 & 8 & 15 & 17 & 19 & 45 & 189216224 \\
6 & 36 & 42 & 5 & 6 & 26 & 27 & 44 & 191157408 \\
\hline
\end{tabular}

* The first solution is due to Subba Rao [28].

For $(6.1 .7), x^{6}=\sum_{1^{7}} y_{i}{ }^{6}$, note that $u^{6} \equiv 0$ or $1(\bmod 8)$ according as $u$ is even or odd. Then for a primitive solution, $x$ and exactly one of the $y_{i}$ are odd. The argument for (6.1.8) modulo 9 applies and $x$ is prime to $6, y_{1}$ (say) is prime to 3 , and either $y_{1}$ is odd or another $y\left(\right.$ say $\left.y_{2}\right)$, is odd. In the first case $y_{1} \equiv \pm x(\bmod 243)$ and $(\bmod 32)$ and $\left(x^{6}-y_{1}^{6}\right) / 6^{6}=\sum_{2}^{7}\left(y_{i} / 6\right)^{6}$ is an integer to be decomposed by trial as the sum of 6 sixth powers. In the second case $y_{1} \equiv \pm x(\bmod 243), y_{2} \equiv \pm x(\bmod$ 32 ) and $\left(x^{6}-y_{1}{ }^{6}-y_{2}{ }^{6}\right) / 6^{6}=\sum_{3}^{7}\left(y_{i} / 6\right)^{6}$ must be an integer (certain combinations $x, y_{1}, y_{2}$ satisfying the congruences are rejected) which is decomposed by trial as the sum of 5 sixth powers. The only solution for $x \leq 1536$ is $(6.1 .7)_{1},(1141)^{6}=$ $(74,234,402,474,702,894,1077)^{6}$ which is obtained in the second case. 
TABLE VI

Primitive solutions of (5. 3. 3) for $z \leq 8 \times 10^{12}$

$$
z=\sum_{1}^{3} x_{j}{ }^{5}=\sum_{1}^{3} y_{j}{ }^{5}
$$

\begin{tabular}{|c|c|c|c|c|c|c|c|}
\hline$i$ & $x_{1}$ & $x_{2}$ & $x_{3}$ & $y_{1}$ & $y_{2}$ & $y_{3}$ & $z$ \\
\hline 1 & 24 & 28 & 67 & 3 & 54 & 62 & 1375298099 \\
\hline 2 & 18 & 44 & 66 & 13 & 51 & 64 & 1419138368 \\
\hline 3 & 21 & 43 & 74 & 8 & 62 & 68 & 2370099168 \\
\hline 4 & 56 & 67 & 83 & 53 & 72 & 81 & 5839897526 \\
\hline$* 5$ & 49 & 75 & 107 & 39 & 92 & 100 & $\begin{array}{llll}1 & 66810 & 39431\end{array}$ \\
\hline 6 & 26 & 85 & 118 & 53 & 90 & 116 & $\begin{array}{lll}2 & 73265 & 12069\end{array}$ \\
\hline 7 & 38 & 47 & 123 & 1 & 89 & 118 & 28461637018 \\
\hline 8 & 73 & 96 & 119 & 68 & 106 & 114 & $\begin{array}{llll}3 & 40903 & 35168\end{array}$ \\
\hline 9 & 39 & 56 & 136 & 3 & 97 & 131 & 47166830151 \\
\hline 10 & 13 & 35 & 142 & 17 & 95 & 138 & $\begin{array}{lll}5 & 77882 & 32400\end{array}$ \\
\hline 11 & 28 & 32 & 155 & 91 & 94 & 150 & $895168 \quad 61675$ \\
\hline 12 & 65 & 94 & 152 & 42 & 129 & 140 & $\begin{array}{lll}8 & 96361 & 42881\end{array}$ \\
\hline 13 & 63 & 67 & 169 & 9 & 131 & 159 & $\begin{array}{lll}14 & 02010 & 53499\end{array}$ \\
\hline 14 & 68 & 137 & 170 & 36 & 140 & 169 & $\begin{array}{ll}19 & 17013 \quad 58025\end{array}$ \\
\hline 15 & 43 & 109 & 181 & 13 & 159 & 161 & $\begin{array}{llll}20 & 97974 & 92893\end{array}$ \\
\hline 16 & 74 & 113 & 182 & 61 & 129 & 179 & $\begin{array}{lll}22 & 03336 & 44849\end{array}$ \\
\hline 17 & 39 & 142 & 186 & 28 & 167 & 172 & $\begin{array}{llll}28 & 04458 & 41607\end{array}$ \\
\hline 18 & 44 & 55 & 201 & 18 & 152 & 190 & $\begin{array}{lll}32 & 87486 & 01600\end{array}$ \\
\hline 19 & 58 & 101 & 204 & 113 & 145 & 195 & $\begin{array}{llll}36 & 44723 & 14293\end{array}$ \\
\hline 20 & 18 & 31 & 215 & 10 & 183 & 191 & 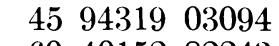 \\
\hline 21 & 19 & 168 & 216 & 11 & 183 & 209 & $\begin{array}{lll}60 & 40152 & 82243\end{array}$ \\
\hline 22 & 5 & 145 & 224 & 153 & 157 & 214 & $6280466 \quad 82374$ \\
\hline 23 & 27 & 106 & 229 & 12 & 122 & 228 & 643159996832 \\
\hline 24 & 151 & 166 & 233 & 126 & 208 & 216 & $\begin{array}{lll}89 & 12718 & 82720\end{array}$ \\
\hline 25 & 59 & 139 & 248 & 23 & 184 & 239 & $99 \quad 0723788966$ \\
\hline 26 & 157 & 193 & 234 & 147 & 218 & 219 & 1064757548174 \\
\hline 27 & 2 & 97 & 258 & 35 & 125 & 257 & $115 \quad 1724993057$ \\
\hline 28 & 3 & 121 & 264 & 163 & 185 & 250 & $\begin{array}{lll}130 & 8325982668\end{array}$ \\
\hline 29 & 97 & 181 & 274 & 67 & 227 & 258 & $17472267 \quad 67782$ \\
\hline 30 & 99 & 105 & 286 & 30 & 179 & 281 & 1935780202300 \\
\hline 31 & 132 & 154 & 283 & 80 & 219 & 270 & 1941923897099 \\
\hline 32 & 106 & 137 & 288 & 201 & 219 & 261 & 2042999635401 \\
\hline 33 & 40 & 168 & 289 & 3 & 215 & 279 & 2149924122017 \\
\hline 34 & 136 & 158 & 294 & 71 & 249 & 268 & $234 \quad 15192 \quad 15168$ \\
\hline 35 & 193 & 229 & 282 & 179 & 259 & 266 & $\begin{array}{llll}268 & 09353 & 50774\end{array}$ \\
\hline 36 & 107 & 229 & 293 & 93 & 259 & 277 & $\begin{array}{llll}280 & 32137 & 94149\end{array}$ \\
\hline 37 & 31 & 173 & 307 & 7 & 201 & 303 & $\begin{array}{llll}288 & 20348 & 39551\end{array}$ \\
\hline 38 & 102 & 118 & 310 & 49 & 270 & 271 & 2896833485600 \\
\hline 39 & 116 & 124 & 310 & 21 & 235 & 294 & $29132347 \quad 67200$ \\
\hline 40 & 30 & 39 & 331 & 65 & 224 & 321 & $39733103 \quad 34850$ \\
\hline 41 & 119 & 232 & 328 & 89 & 289 & 301 & 4492348861399 \\
\hline 42 & 108 & 181 & 348 & 53 & 246 & 338 & $\begin{array}{llll}531 & 27877 & 53637\end{array}$ \\
\hline 43 & 114 & 211 & 364 & 52 & 298 & 339 & $\begin{array}{lll}682 & 75705 & 13699\end{array}$ \\
\hline 44 & 172 & 206 & 364 & 102 & 303 & 337 & $\begin{array}{lll}691 & 15935 & 15232\end{array}$ \\
\hline 45 & 123 & 137 & 373 & 13 & 259 & 361 & $\begin{array}{lll}729 & 65305 & 14393\end{array}$ \\
\hline
\end{tabular}

* This solution was found by A. Moessner [35]. 
TABLE VII

(k. m. n $)_{1}$ and summary of results

Range

(k. $m . n) \quad$ Searched Solutions Known*

\begin{tabular}{lll}
\hline 4.1 .3 & $2.34 \times 10^{21}$ & None known \\
4.1 .4 & $4.1 \times 10^{15}$ & $(353)^{4}=(30,120,272,315)^{4}$ \\
& & See Table I, 23 solutions
\end{tabular}

4. 1.5

4. 2.2

$5.3 \times 10^{16}$

4. 2.3

4. 3.3

5. 1.3

5. 1.4

5. 1.5

$2.6 \times 10^{14}$

$2.6 \times 10^{14}$

$(5)^{4}=\left(2^{2}, 3,4^{2}\right)^{4}$

$(59,158)^{4}=(133,134)^{4}$

See Table I in [18], and Table II, 46 solutions

$\left(7^{2}\right)^{4}=(3,5,8)^{4}$

$(2,4,7)^{4}=\left(3,6^{2}\right)^{4}$

5. 1.6

5. 1.7

5. 2.2

5. 2.3

5. 2.4

$7.7 \times 10^{13}$

5. 2.5

$2.8 \times 10^{14}$
$8 \times 10^{12}$
$2 \times 10^{10}$

None known

$(144)^{5}=(27,84,110,133)^{5}$

$(72)^{5}=(19,43,46,47,67)^{5}$

See Table III, 12 solutions

$(12)^{5}=(4,5,6,7,9,11)^{5}$

$(23)^{5}=(1,7,8,14,15,18,20)^{5}$

5. 3.3

$2 \times 10^{8}$

5. 3.4

5. 4.4

6. $1 . n$

6. 1.7

$8 \times 10^{12}$

6. 1.8

$3.16 \times 10^{27}$

None known

None known

$(3,29)^{5}=(4,10,20,28)^{5}$

See Table IV, 10 solutions

$(1,22)^{5}=(4,5,7,16,21)^{5}$

See Table V, 6 solutions

$(24,28,67)^{5}=(3,54,62)^{5}$

See Table VI, 45 solutions

$(3,22,25)^{5}=(1,8,14,27)^{5}$

$\left(5,6^{2}, 8\right)^{5}=\left(4,7^{3}\right)^{5}$

$1.3 \times 10^{19}$

None known for $n \leq 6$

$(1141)^{6}=(74,234,402,474,702,894,1077)^{6}$

$5.8 \times 10^{16}$

6. 1.9

6. 1.10

6. 1.11

6. 2. $n$

6. 2.7

6. 2.8

6. 2.9

6. 2.10

6. 3.3

$2.5 \times 10^{14}$

6. 3. 4

$2.9 \times 10^{12}$

6. 4.4

7. $1 . n$

$4 \times 10^{12}$

$(251)^{6}=(8,12,30,78,102,138,165,246)^{6}$

See Table VIII, 14 solutions

$(54)^{6}=\left(1,17,19,22,31,37^{2}, 41,49\right)^{6}$

$(39)^{6}=\left(2,4,7,14,16,26^{2}, 30,32^{2}\right)^{6}$

$(18)^{6}=\left(2,5^{3}, 7^{2}, 9^{2}, 10,14,17\right)^{6}$

None known for $n \leq 6$

$(56,91)^{6}=\left(18,22,36,58,69,78^{2}\right)^{6}$

$(35,37)^{6}=(8,10,12,15,24,30,33,36)^{6}$

$(6,21)^{6}=\left(1,5^{2}, 7,13^{3}, 17,19\right)^{6}$

$\left(12^{2}\right)^{6}=\left(1^{3}, 4^{2}, 7,9,11^{3}\right)^{6}$

$(3,19,22)^{6}=(10,15,23)^{6}$

See Table IX, 10 solutions

$(41,58,73)^{6}=(15,32,65,70)^{6}$

See Table $X, 5$ solutions

$\left(2^{2}, 9^{2}\right)^{6}=(3,5,6,10)^{6}$
None known for $n \leq 7$

7. 1.8

$1.95 \times 10^{14}$

$(102)^{7}=(12,35,53,58,64,83,85,90)^{7}$

$(62)^{7}=(6,14,20,22,27,33,41,50,59)^{7}$

$(10,33)^{7}=\left(5,6,7,15^{2}, 20,28,31\right)^{7}$

$\left(26,30^{2}\right)^{7}=\left(7^{2}, 12,16,27,28,31\right)^{7}$

$(12,16,43,50)^{7}=(3,11,26,29,52)^{7}$

$\left(8^{2}, 13,16,19\right)^{7}=(2,12,15,17,18)^{7}$

See Table XI, 17 solutions

* All solutions shown are (k. m. n $)_{1}$ unless otherwise marked. 


\section{TABLE VII (cont.)}

\begin{tabular}{|c|c|c|}
\hline$(k \cdot m \cdot n)$ & $\begin{array}{l}\text { Range } \\
\text { Searched }\end{array}$ & Solutions Known \\
\hline 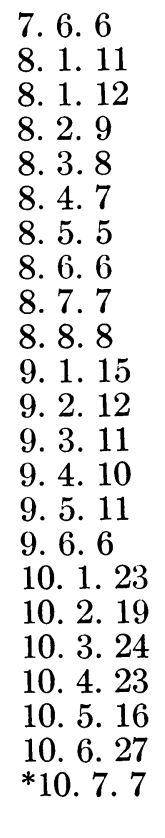 & & $\begin{array}{l}\left(2,3,6^{2}, 10,13\right)^{7}=\left(1^{2}, 7^{2}, 12^{2}\right)^{7} \\
(125)^{8}=\left(14,18,44^{2}, 66,70,92,93,96,106,112\right)^{8} \\
(65)^{8}=\left(8^{2}, 10,24^{3}, 26,30,34,44,52,63\right)^{8} \\
(11,27)^{8}=\left(2,7,8,16,17,20^{2}, 24^{2}\right)^{8} \\
(8,17,50)^{8}=\left(6,12,16^{2}, 38^{2}, 40,47\right)^{8} \\
(6,11,20,35)^{8}=\left(7,9,16,22^{2}, 28,34\right)^{8} \\
(1,10,11,20,43)^{8}=(5,28,32,35,41)^{8} \\
(3,6,8,10,15,23)^{8}=\left(5,9^{2}, 12,20,22\right)^{8} \\
\left(1,3,5,6^{2}, 8,13\right)^{8}=\left(4,7,9^{2}, 10,11,12\right)^{8} \\
\left(1,3,7^{3}, 10^{2}, 12\right)^{8}=\left(4,5^{2}, 6^{2}, 11^{3}\right)^{8} \\
(26)^{9}=\left(2^{2}, 4,6^{2}, 7,9^{2}, 10,15,18,21^{2}, 23^{2}\right)^{9} \\
(15,21)^{9}=\left(2^{4}, 3^{2}, 4,7,16,17,19^{2}\right)^{9} \\
(13,16,30)^{9}=\left(2,3,6,7,9^{2}, 19^{2}, 21,25,29\right)^{9} \\
(5,12,16,21)^{9}=\left(2,6^{2}, 9,10,11,14,18,19^{2}\right)^{9} \\
(7,8,14,20,22)^{9}=\left(3,5^{2}, 9^{2}, 12,15^{2}, 16,21^{2}\right)^{9} \\
\left(1,13^{2}, 14,18,23\right)^{9}=(5,9,10,15,21,22)^{9} \\
(15)^{10}=\left(1^{5}, 2,3,6,77^{6}, 10,12^{4}, 13,14\right)^{10} \\
(9,17)^{10}=\left(2^{5}, 5,6,10,11^{6}, 12^{2}, 15^{3}\right)^{10} \\
\left(11,15^{2}\right)^{10}=\left(1,2,3,4^{10}, 7,8^{7}, 10,12,16\right)^{10} \\
\left(11^{3}, 16\right)^{10}=\left(1^{5}, 2^{2}, 3^{2}, 4,6^{4}, 7^{3}, 8,10^{2}, 14^{2}, 15\right)^{10} \\
\left(3^{2}, 8,14,16\right)^{10}=\left(1^{4}, 2,4^{2}, 6,12^{2}, 13^{5}, 15\right)^{10} \\
\left(2^{2}, 8,11,12^{2}\right)^{10}=\left(1,3^{4}, 4^{2}, 5^{2}, 6^{7}, 7^{9}, 10,13\right)^{10} \\
(1,28,31,32,55,61,68)^{10}=(17,20,23,44,49,64, \\
\quad 67)^{10}\end{array}$ \\
\hline
\end{tabular}

* Moessner [35]; not known to be (10.7. 7) 1 .

For $(6.1 .6), x^{6}=\sum_{1}^{6} y_{i}{ }^{6}$ note that $u^{6} \equiv 0$ or $1(\bmod 7)$ according as $u \equiv 0$ or $u \not \equiv 0(\bmod 7)$. Then for a primitive solution, $x$ and exactly one of the $y_{i}$ (say $\left.y_{1}\right)$ are prime to 7 . This implies $y_{1} \equiv \pm x, \pm q x$ or $\pm q^{2} x$ where $q=34968$ is a primitive sixth root of unity $\left(\bmod 7^{6}=117649\right)$. Now the foregoing arguments modulo 8 and modulo 9 apply, and there are five cases.

(1) If $y_{1} \equiv \pm 1(\bmod 6)$ then $y_{1} \equiv \pm x(\bmod 243)$ and $(\bmod 32)$ and $\left(x^{6}-y_{1}{ }^{6}\right) / 42^{6}$ $=\sum_{2}^{6}\left(y_{i} / 42\right)^{6}$ is an integer to be decomposed by trial as the sum of 5 sixth powers.

(2) If $y_{1} \equiv \pm 2(\bmod 6)$ then $y_{1} \equiv \pm x(\bmod 243)$ and another of the $y_{i}\left(\operatorname{say} y_{2}\right)$, is odd. Then $y_{2} \equiv 0(\bmod 3 \cdot 7), y_{2} \equiv \pm x(\bmod 32)$, and $\left(x^{6}-y_{1}{ }^{6}-y_{2}{ }^{6}\right) / 42^{6}=$ $\sum_{3}^{6}\left(y_{i} / 42\right)^{6}$ is the sum of 4 integral sixth powers.

(3) If $y_{1} \equiv 3(\bmod 6)$ then $y_{1} \equiv \pm x(\bmod 32)$ and another of the $y_{i}\left(\operatorname{say} y_{2}\right)$, is prime to $3, y_{2} \equiv 0(\bmod 2 \cdot 7)$, and $y_{2} \equiv \pm x(\bmod 243)$. In case $(2),\left(x^{6}-y_{1}{ }^{6}-y_{2}{ }^{6}\right) / 42^{6}$ is an integer and is the sum of 4 sixth powers.

(4) If $y_{1} \equiv 0(\bmod 6)$, another of the $y_{i}\left(\operatorname{say} y_{2}\right)$, is prime to $3, y_{2} \equiv 0(\bmod 7)$ and $y_{2} \equiv \pm x(\bmod 243)$. If $y_{2}$ is odd, then $y_{2} \equiv \pm x(\bmod 32)$ and as in cases $(2)$ and (3) $\left(x^{6}-y_{1}{ }^{6}-y_{2}{ }^{6}\right) / 42^{6}$ is the sum of 4 sixth powers. If $y_{2}$ is even, we have case (5).

(5) Another of the $y_{i}\left(\operatorname{say} y_{3}\right)$, is odd, $y_{3} \equiv 0(\bmod 3 \cdot 7), y_{3} \equiv \pm x(\bmod 32)$, and $\left(x^{6}-y_{1}{ }^{6}-y_{2}{ }^{6}-y_{3}{ }^{6}\right) / 42^{6}=\sum_{4}^{6}\left(y_{i} / 42\right)^{6}$ is an integer to be decomposed as the sum of 3 sixth powers. 
The search for a solution to (6. 1.6) was carried exhaustively by this method through the range $x \leq 38314$ and there is no solution in this range.

A. Martin [30] gave a solution to (6. 1. 16); Moessner [31] gave solutions to (6. $1 . n)$ for $n=16,18,20$ and 23. For $n \geq 11$, it is not difficult to find solutions in small integers.

TABLE VIII

Primitive Solutions of (6. 1.8) for $z \leq 7 \times 10^{16}$ $z=x_{1}{ }^{6}=\sum_{1}^{8} y_{i}{ }^{6}$

\begin{tabular}{|c|c|c|c|c|c|c|c|c|c|}
\hline$i$ & $x_{1}$ & $y_{1}$ & $y_{2}$ & $y_{3}$ & $y_{4}$ & $y_{5}$ & $y_{6}$ & $y_{7}$ & $y_{8}$ \\
\hline 1 & 251 & 8 & 12 & 30 & 78 & 102 & 138 & 165 & 246 \\
\hline 2 & 431 & 48 & 111 & $1 \check{6} 6$ & 186 & 188 & 228 & 240 & 426 \\
\hline 3 & 440 & 93 & 93 & 195 & 197 & 303 & 303 & 303 & 411 \\
\hline 4 & 440 & 219 & $25 \check{5}$ & 261 & 267 & 289 & 351 & 351 & 351 \\
\hline 5 & 455 & 12 & 66 & 138 & 174 & 212 & 288 & 306 & 441 \\
\hline 6 & 493 & 12 & 48 & 222 & 236 & 333 & 384 & 390 & 426 \\
\hline 7 & 499 & 66 & 78 & 144 & 228 & 256 & 288 & 435 & 444 \\
\hline 8 & 502 & 16 & 24 & 60 & 156 & 204 & 276 & 330 & 492 \\
\hline 9 & 547 & 61 & 96 & 156 & 228 & 276 & 318 & 354 & 534 \\
\hline 10 & 559 & 170 & 177 & 276 & 312 & 312 & 408 & 450 & 498 \\
\hline 11 & 581 & 60 & 102 & 126 & 261 & 270 & 338 & 354 & 570 \\
\hline 12 & 583 & 57 & 146 & 150 & 360 & 390 & 402 & 444 & 528 \\
\hline 13 & 607 & 33 & 72 & 122 & 192 & 204 & 390 & 534 & 534 \\
\hline 14 & 623 & 12 & 90 & 114 & 114 & 273 & 306 & 492 & 592 \\
\hline
\end{tabular}

(6. 3. n)-Subba Rao [32] found the solution $(3,19,22)^{6}=(10,15,23)^{6}$ which is (6. 3. 3) 1 . In Table IX we give the remaining 9 primitive solutions which exist in the range up to $2.5 \times 10^{14}$. It is interesting to note that each of the solutions except the sixth is also a solution to (2.3.3). Table $\mathrm{X}$ gives the five primitive solutions to (6. 3. 4) which exist in the range up to $2.9 \times 10^{12}$.

(6. $m$. n)-If $m$ is $\geq 4$, solutions in small integers can be found readily. Subba Rao [32] gave (6.4.4) (see Table VII). The first triple coincidence of 4 sixth powers is $1885800643779=(1,34,49,111)^{6}=(7,43,69,110)^{6}=(18,25,77,109)^{6}$.

\section{Seventh Powers.}

$(7.2 .10)_{2}$ is $(2,27)^{7}=\left(4,8,13,14^{2}, 16,18,22,23^{2}\right)^{7}=\left(7^{2}, 9,13,14,18,20,22^{2}, 23\right)^{7}$ which is a double primitive and reduces to the solution $(7.5 .5)_{2}$. 


\section{TABLE IX}

Primitive solutions of (6. 3. 3) for $z \leq 2.5 \times 10^{14}$

$$
z=\sum_{1}^{3} x_{j}{ }^{6}=\sum_{1} y_{j}{ }^{6}
$$

\begin{tabular}{|c|c|c|c|c|c|c|c|}
\hline$i$ & $x_{1}$ & $x_{2}$ & $x_{3}$ & $y_{1}$ & $y_{2}$ & $y_{3}$ & $z$ \\
\hline$*_{1}$ & 3 & 19 & 22 & 10 & 15 & 23 & 160426514 \\
\hline 2 & 36 & 37 & 67 & 15 & 52 & 65 & 95200890914 \\
\hline 3 & 33 & 47 & 74 & 23 & 54 & 73 & $17 \quad 62771 \quad 73474$ \\
\hline 4 & 32 & 43 & 81 & 3 & 55 & 80 & $2898246 \quad 41354$ \\
\hline j & 37 & 50 & 81 & 11 & 65 & 78 & $\begin{array}{lll}30 & 06202 & 62890\end{array}$ \\
\hline 6 & 25 & 62 & 138 & 82 & 92 & 135 & $\begin{array}{lll}696 & 38068 & 13393\end{array}$ \\
\hline 7 & 51 & 113 & 136 & 40 & 125 & 129 & 8427066928346 \\
\hline 8 & 71 & 92 & 147 & 1 & 132 & 133 & $108247536 \quad 54794$ \\
\hline 9 & 111 & 121 & 230 & 26 & 169 & 225 & 153044731928882 \\
\hline 10 & 75 & 142 & 245 & 14 & 163 & 243 & $22464 \quad 6509202194$ \\
\hline
\end{tabular}

* The first solution is due to K. Subba Rao [32].

TABLE X

Primitive solutions of (6. 3. 4) for $z \leq 2.9 \times 10^{12}$

$$
z=\sum_{1}^{3} x_{j}{ }^{6}=\sum_{1}^{4} y_{j}{ }^{6}
$$

\begin{tabular}{r|rll|llll|rl}
\hline$i$ & $x_{1}$ & $x_{2}$ & $x_{3}$ & $y_{1}$ & $y_{2}$ & $y_{3}$ & $y_{4}$ & \multicolumn{2}{|c}{$z$} \\
\hline 1 & 41 & 58 & 73 & 15 & 32 & 65 & 70 & 194153023074 \\
2 & 61 & 62 & 85 & 52 & 56 & 69 & 83 & 485470125570 \\
3 & 61 & 74 & 85 & 26 & 56 & 71 & 87 & 592876380162 \\
4 & 11 & 88 & 90 & 21 & 74 & 78 & 92 & 995846858345 \\
5 & 26 & 83 & 95 & 23 & 24 & 28 & 101 & 1062341179770 \\
\hline
\end{tabular}

(7. 5. n)-Table XI lists the 17 primitive solutions to (7.5.5) which exist in the range up to $4.0 \times 10^{12}$.

\section{Eighth Powers.}

(8. 1. $n)$-We found a parametric solution to $(8.1 .17),\left(2^{8 k+4}+1\right)^{8}=\left(2^{8 k+4}-1\right)^{8}$ $+\left(2^{7 k+4}\right)^{8}+\left(2^{k+1}\right)^{8}+7\left[\left(2^{5 k+3}\right)^{8}+\left(2^{3 k+2}\right)^{8}\right]$ which for $k=0$ yields $(8.1$. 17) 1 . This was the solution used by Sastry [26] in developing a parametric solution to (8. 8. 8). The computer program used in searching for solutions to (8. 1. $n$ ) was based on the congruences $x^{8} \equiv 0$ or $1(\bmod 32)$ according as $x \equiv 0$ or $1(\bmod 2)$ so that primitive solutions to $x^{8}=\sum_{1}^{n} y_{j}^{8}$ with $n<32$ must have $x$ and (say) $y_{1}$ both odd. Then $x^{8}-$ $y_{1}^{8}$ is divisible by $2^{8}$ which implies $x \equiv \pm y_{1}(\bmod 32)$, and $\left(x^{8}-y_{1}^{8}\right) / 256$ is decomposed as the sum of $n-1$ eighth powers by trial.

Solutions to (8. 5. 5) and (8.9.9) were found by A. Letac [33]. 
Ninth and Tenth Powers. Computations performed by the authors for (9.m.n) and (10.m.n) are the basis for the data shown in the last two columns of Table XII,

TABLE XI

Primitive solutions of (7. 5. 5) for $z \leq 4.0 \times 10^{12}$

$z=\sum_{1}^{5} x_{j}{ }^{7}=\sum_{1}^{5} y_{j}{ }^{7}$

\begin{tabular}{r|rrlll|rrrrr|rrrr}
\hline$i$ & $x_{1}$ & $x_{2}$ & $x_{3}$ & $x_{4}$ & $x_{5}$ & $y_{1}$ & $y_{2}$ & $y_{3}$ & $y_{4}$ & $y_{5}$ & & & & \\
\hline 1 & 8 & 8 & 13 & 16 & 19 & 2 & 12 & 15 & 17 & 18 & & 12292 & 50016 \\
2 & 4 & 8 & 14 & 16 & 23 & 7 & 7 & 9 & 20 & 22 & & 37807 & 87943 \\
3 & 11 & 12 & 18 & 21 & 26 & 9 & 10 & 22 & 23 & 24 & 1 & 05004 & 37728 \\
4 & 6 & 12 & 20 & 22 & 27 & 10 & 13 & 13 & 25 & 26 & 1 & 42708 & 22835 \\
5 & 3 & 13 & 17 & 24 & 38 & 14 & 26 & 32 & 32 & 33 & 11 & 94751 & 43393 \\
6 & 4 & 5 & 30 & 36 & 44 & 2 & 8 & 27 & 39 & 43 & 41 & 95120 & 68269 \\
7 & 16 & 33 & 33 & 33 & 44 & 18 & 26 & 34 & 38 & 43 & 44 & 74015 & 74051 \\
8 & 3 & 4 & 21 & 39 & 45 & 14 & 23 & 33 & 41 & 43 & 51 & 27015 & 66916 \\
9 & 16 & 17 & 26 & 33 & 49 & 10 & 12 & 30 & 43 & 46 & 72 & 95521 & 00131 \\
10 & 15 & 18 & 18 & 43 & 48 & 8 & 11 & 32 & 44 & 47 & 86 & 02822 & 52818 \\
11 & 19 & 24 & 43 & 46 & 51 & 9 & 36 & 40 & 48 & 50 & 161 & 05272 & 89337 \\
12 & 13 & 16 & 35 & 35 & 56 & 9 & 19 & 28 & 44 & 55 & 185 & 61046 & 27259 \\
13 & 9 & 11 & 43 & 45 & 55 & 3 & 19 & 37 & 51 & 53 & 216 & 79475 & 68747 \\
14 & 9 & 15 & 19 & 34 & 59 & 5 & 10 & 16 & 48 & 57 & 254 & 22443 & 49046 \\
15 & 23 & 27 & 40 & 49 & 56 & 7 & 39 & 45 & 51 & 53 & 258 & 30231 & 01035 \\
16 & 8 & 13 & 41 & 45 & 59 & 2 & 10 & 47 & 52 & 55 & 305 & 71400 & 57494 \\
17 & 1 & 38 & 39 & 39 & 60 & 8 & 25 & 34 & 53 & 57 & 318 & 82375 & 95951 \\
\hline
\end{tabular}

TABLE XII

Least $n$ for which a solution to (k. m. n) is known

\begin{tabular}{c|rrrrrrrrr}
\hline & \multicolumn{10}{|c}{$k$} \\
\cline { 2 - 9 }$m$ & 2 & 3 & 4 & 5 & 6 & 7 & 8 & 9 & 10 \\
\hline 1 & 2 & 3 & 4 & 4 & 7 & 8 & 11 & 15 & 23 \\
2 & 2 & 2 & 2 & 4 & 7 & 8 & 9 & 12 & 19 \\
3 & & & & 3 & 3 & 7 & 8 & 11 & 24 \\
4 & & & & & & 5 & 7 & 10 & 23 \\
5 & & & & & & 5 & 5 & 11 & 16 \\
6 & & & & & & & & 6 & 27 \\
7 & & & & & & & & & 7 \\
\hline
\end{tabular}

except for a solution to (10.7.7) given by A. Moessner [35]. Due to computer word length limitations the calculations were not extended to large values of the arguments.

Additional References. A. Gloden gave a parametric solution of (5. 4. 4) in [38], two parametric solutions of (7. 5. 5) in [39], [40], and a parametric solution of (8. 7. 7) in [41]. A. Moessner gave numerical solutions of (5. 2.4) and (5. 3. 3) in [42]. In [43] Moessner gave three parametric solutions of (6. 4. 4) and parametric solutions of (8. 7. 7) and (9. 10.10). Two numerical solutions of (7. 4. 5) due to A. Letac are found in [39]. S. Sastry and T. Rai solved (7.6.6) parametrically [44]. 
G. Palamà [45] gave numerical solutions of $(9.11 .11)$ and (11. 10. 12). In [46] Moessner and Gloden solved (8.6.6) and (8.6.7) numerically.

Concluding Remarks. Let $N(k, m)$ be the smallest $n$ for which (k.m.n) is solvable. In Table XII we show the upper bound to $N$ based on the results just presented. Each column is terminated when a solution to $(k . m . m)$ has been found. It appears likely that whenever $(k . m . m)$ is solvable, so is $(k . r . r)$ for any $r>m$. Some questions are:

(a) Is $N(k, m+1) \leq N(k, m) \leq N(k+1, m)$ always true?

(b) Is $(k, m . n)$ always solvable when $m+n>k$ ?

(c) Is it true that (k.m.n) is never solvable when $m+n<k$ ?

(d) For which $k, m, n$ such that $m+n=k$ is ( $k . m$. $n$ ) solvable?

The results presented in this paper tend to support an affirmative answer to (c). Question (d) appears to be especially difficult. The only solvable cases with $m+n$ $=k$ known at present are (4.2.2), (5.1.4) and (6.3.3).

In this paper we have made a computational attack on the problem of finding a sum of $n k$ th powers which is also the sum of a smaller number of $k$ th powers. In many of the cases considered, especially for the larger values of $k$, we have undoubtedly not obtained the best possible results, but the amount of computing needed to do this would seem to be overwhelming.

We believe that the main result of this paper is the presentation of results on a family of Diophantine equations which have largely been considered separately in the past. We hope that this presentation offers greater insight into the nature of the function $N(k, m)$ and that future efforts will be directed toward reducing the upper bounds for this function.

Aerospace Corporation

Los Angeles, California 90045

Aerospace Corporation

Los Angeles, California 90045

Pennsylvania State University

University Park, Pennsylvania 16802

1. G. H. HARDy \& E. M. Wright, An Introduction to the Theory of Numbers, 3rd ed., Clarendon Press, Oxford, 1954; 4th ed., pp. 332-335, Oxford Univ. Press, London, 1960. MR $16,673$.

2. L. E. Dickson, History of the Theory of Numbers, Vol. 2, chapters 4-9, 21-24, Publication No. 256, Carnegie Institution of Washington, Washington, D. C., 1920; reprint, Stechert, New York, 1934.

3. L. E. Dickson, ibid., pp. 165-170.

4. L. E. DICKSON, ibid., p. 252.

5. J. L. Selfridge \& B. W. Pollack, Notices Amer. Math. Soc., no. 11, 1964, p. 97.

6. L. E. Dickson, loc. cit., pp. 550-554.

7. L. E. Dickson, loc. cit., pp. 563-565.

8. M. WARD, "Euler's problem on sums of three fourth powers," Duke Math J., v. 15, 1948, pp. 827-837. MR 10,283.

9. R. Norrie, University of St. Andrews 500th Anniversary Memorial Volume, Edinburgh, 1911, pp. 87-89.

10. J. LeECH, "On $A^{4}+B^{4}+C^{4}+D^{4}=E^{4}$," Proc. Cambridge Philos. Soc., v. 54, 1958, pp. 554-555. MR 20 \#2301.

11. S. Brudvo, "A further example of $A^{4}+B^{4}+C^{4}+D^{4}=E^{4}$," Proc. Cambridge Philos. Soc., v. 60, 1964, pp. 1027-1028. MR $29 \# 3429$.

12. E. Fauquembergue, L'intermédiaire des Math., v. 5, 1898, p. 33.

13. C. Haldeman, Math. Mag., v. 2, 1904, pp. 288-296. 
14. A. Martin, Deux. Congrès Internat. Math., 1900, Paris, 1902, pp. 239-248; reproduced with additions in Math. Mag., v. 2, 1910, pp. 324-352.

15. L. Euler, Nova Acta Acad. Petrop., 13, ad annos 1795-1796, 1802 (1778), 45; Comm. Arith.,

II, 281. Cited in L. E. Dickson, loc. cit., pp. 645-646.

16. A. GÉrardin, L'intermédiaire des Math., v. 24, 1917, p. 51.

17. J. LeEch, "Some solutions of Diophantine equations," Proc. Cambridge Philos. Soc., v. 53, 1957, pp. 778-780. MR 19, 837.

18. L. Lander \& T. Parkin, "Equal sums of biquadrates," Math. Comp., v. 20, 1966, pp. 450-451.

19. A. GÉrardin, Assoc. franç., v. 39, 1910, I, pp. 44-55; same in Sphinx-Oedipe, v. 5, 1910,

pp. $180-186$; v. 6,1911 , pp. $3-6$; v. 8,1913 , p. 119 .

20. F. Ferrari, L'intermédiaire des Math., v. 20, 1913, pp. 105-106.

21. A. GÉrardin, Bull. Soc. Philomathique, (10), v. 3, 1911, p. 236.

22. A. Werebrusow, L'intermédiaire des Math., v. 20, 1913, pp. 105-106.

23. L. Lander \& T. Parkin, "A counterexample to Euler's conjecture on like powers," Bull. Amer. Math. Soc., v. 72, 1966, p. 173.

24. L. LANDER \& T. PARKin, "A counterexample to Euler's sum of powers conjecture," Math. Comp., v. 21, 1967, pp. 101-103.

25. L. E. Dickson, loc. cit., p. 648.

26. S. SASTRY, "On sums of powers," J. London Math. Soc., v. 9, 1934, pp. 242-246.

27. A. Martin, Bull. Philos. Soc. Wash., v. 10, 1887, p. 107, in Smithsonian Miscel. Coll., v. 33,1888 .

28. K. Subba RaO, "On sums of fifth powers," J. London Math. Soc., v. 9, 1934, pp. 170-171.

29. G. Xeroudakes \& A. Moessner, "On equal sums of like powers," Proc. Indian Acad. Sci. Sect. A, v. 48, 1958, pp. 245-255. MR 21 \#20.

30. A. Martin, Quart. J. Math., v. 26, 1893, pp. 225-227.

31. A. Momssner, "Einige zahlentheoretische Untersuchungen und diophantische Probleme,"

Glasnik Mat.-Fiz. Astronom. Drustvo Mat. Fiz. Hrvatske Ser. II, v. 14, 1959, pp. 177-182. MR 24 \#A2558.

32. K. Subba Rao, “On sums of sixth powers," loc. cit., pp. 172-173.

33. A. Letac, Gazeta Matematica, v. 48, 1942, pp. 68-69.

34. J. O. Patterson, "A note on the Diophantine problem of finding four biquadrates whose sum is a biquadrate," Bull. Amer. Math. Soc., v. 48, 1942, pp. 736-737. MR 4,33.

35. A. Momssner, "Einige numerische Identitäten," Proc. Indian Acad. Sci. Sect. A, v. 10, 1939, pp. 296-306. MR 1, 133.

36. H. P. F. Swinnerton-Dyer, "A solution of $A^{5}+B^{5}+C^{5}=D^{5}+E^{5}+F^{5}$," Proc. Cambridge Philos. Soc., v. 48, 1952, pp. 516-518. MR 13, 913.

37. L. E. Dickson, loc. cit., p. 644. 11,82 .

38. A. Gloden, "Über mehrgradige Gleichungen," Arch. Math., v. 1, 1949, pp. 482-483. MIR

39. A. Gloden, "Sur la multigrade $A_{1} A_{2}, A_{3}, A_{4}, A_{5}{ }^{k}=B_{1}, B_{2}, B_{3}, B_{4}, B_{5}(k=1,3,5,7)$," Revista Euclides, v. 8, 1948, pp. 383-384. MR 10, 431.

40. A. Gloden, "Zwei Parameterlösungen einer mehrgradigen Gleichung," Arch. Math., v. 1,1949 , pp. $480-482$. MR 11,82 .

41. A. Gloden, "Parametric solutions of two multi-degreed equalities," Amer. Math. Monthly, v. 55,1948 , pp. 86-88. MR 9, 331.

42. A. Moessner, "Alcune richerche di teoria dei numeri e problemi diofantei," Bol. Soc. Mat. Mexicana (5), v. 2, 1948, pp. 36-39. MR 10, 592 .

43. A. Moessner, "On equal sums of like powers," Math. Student, v. 15, 1947, pp. 83-88. MR 11, 500 .

44. S. SaStry \& T. RaI, "On equal sums of like powers," Math. Student, v. 16, 1948, pp. 18-19. MR 11, 391.

45. G. PALAmì, "Diophantine systems of the type $\sum_{i=1}^{p} a_{i}{ }^{k}=\sum_{i=1}^{p} b_{i}{ }^{k},(k=1,2, \cdots, n, n+2$, $n+4, \cdots, n+2 r)$," Scripta Math., v. 19, 1953, pp. 132-134. MR 15, 199.

46. A. Moessner \& A. Gloden, "Einige Zahlentheoretische Untersuchungen und Resultate," Bull. Sci. École Polytech. de Timişoara, v. 11, 1944, pp. 196-219. MR 9, 9. 